\title{
Effectiveness of the Novel Herbal Medicine, KIOM-MA, and Its Bioconversion Product, KIOM-MA128, on the Treatment of Atopic Dermatitis
}

\author{
Tae Ho Chung, ${ }^{1}$ Tae Jin Kang, ${ }^{2}$ Won-Kyung Cho, ${ }^{1}$ Ga Young Im, ${ }^{1}$ Geum Seon Lee, \\ Min Cheol Yang, ${ }^{1}$ Chang-Won Cho, ${ }^{3}$ and Jin Yeul $\mathrm{Ma}^{1}$ \\ ${ }^{1}$ Center for Herbal Medicine Improvement Research, Korea Institute of Oriental Medicine (KIOM), 483 Expo-ro, Yuseong-gu, \\ Daejeon 305-811, Republic of Korea \\ ${ }^{2}$ College of Pharmacy, Sahmyook University, 26-21 Kongnung 2-dong, Nowon-gu, Seoul 139-742, Republic of Korea \\ ${ }^{3}$ Regional Food Industry Research Group, Korea Food Research Institute, Sungnam 463-746, Republic of Korea
}

Correspondence should be addressed to Jin Yeul Ma, jyma@kiom.re.kr

Received 23 August 2011; Accepted 10 October 2011

Academic Editor: Olumayokun A. Olajide

Copyright (C) 2012 Tae Ho Chung et al. This is an open access article distributed under the Creative Commons Attribution License, which permits unrestricted use, distribution, and reproduction in any medium, provided the original work is properly cited.

\begin{abstract}
This study was conducted to determine if oral administration of the novel herbal medicine, KIOM-MA, and its Lactobacillus acidophilus-fermented product, KIOM-MA128, has therapeutic properties for the treatment of atopic dermatitis (AD). Using ADinduced BALB/c mice by Ovalbumin and aluminum hydroxide, the effectiveness of KIOM-MA and KIOM-MA128 on AD was evaluated. Oral administration of KIOM-MA and KIOM-MA128 reduced major clinical signs of AD including erythema/darkening, edema/papulation, excoriations, lichenification/prurigo, and dryness. Interestingly, KIOM-MA128 more significantly improved $\mathrm{AD}$-related symptoms including decrease of IgE level in the plasma as well as reduction of scratching behavior, skin severity in the AD BALB/c model. HPLC analysis showed the significant changes in the constituent patterns between KIOM-MA and KIOMMA128. Our results suggest that both KIOM-MA and KIOM-MA128 have potential for therapeutic reagent for the treatment of $\mathrm{AD}$, and further, the efficacy is significantly enhanced by L. acidophilus fermentation via increases in its indicator molecule.
\end{abstract}

\section{Introduction}

Atopic dermatitis (AD) is a chronic inflammatory skin disease associated with cutaneous hyperreactivity to environmental triggers that are innocuous to normal nonatopic individuals [1]. Various studies have shown that atopic dermatitis has a complex etiology, with activation of multiple immunological and inflammatory pathways [2]. In addition, several studies have suggested that atopic dermatitis is the cutaneous manifestation of a systemic disorder that also gives rise to asthma, food allergy, and allergic rhinitis $[3,4]$. These conditions are all characterized by elevated serum IgE levels and peripheral eosinophilia [5].

$\mathrm{AD}$ has long been considered a systemic disease for which there are few satisfactory systemic therapies that do not involve glucocorticoids [6]. In general, topically applied medication of adequate potency, mainly corticosteroids, has been standard for the vast majority of patients with $\mathrm{AD}[6]$.
Although intermittent use of topical corticosteroids is highly effective, the repeated use of steroids is not desirable due to the systemic adverse effects their long-term use can induce $[7,8]$. Accordingly, research has begun to identify steroidfree alternative therapeutic agents with better efficacy and safety [9].

There has been increased interest in the use of traditional herbal medicine to develop new therapeutic agents without a steroid for $\mathrm{AD}$ treatment [10]. In addition, recent double-blind, placebo-controlled, crossover studies show considerably effective benefits in managing clinical signs of $\mathrm{AD}$ [11-14]. However, it is important to investigate the active principles of the herbal medicines for quality control and to determine their real therapeutic value in modern pharmacology $[15,16]$. Recent studies have suggested that fermentation of herbal extracts may affect the therapeutic potential due to their increased absorptive effect [17]. 
In this study, we report that newly formulated herbal medicine KIOM-MA possesses strong antiatopic activity and KIOM-MA128, fermented form by L. acidophilus, exerts more potent inhibitory effect on AD.

\section{Materials and Methods}

2.1. Preparation and Fermentation of Novel Herbal Medicine. To develop a novel herbal medicine formula for the treatment of $\mathrm{AD}$, over 100 herbal medical formulas were tested by the Center for Herbal Medicine Improvement Research at the Korea Institute of Oriental Medicine. A novel herbal medicine with antiatopic dermatitis effects, KIOM-MA (Glycyrrhizae Radix, Polygoni Cuspidati Rhizoma, Sophorae Radix, Cnidii Rhizoma, Arctii Fructus, etc., Korean Patent Application no. 10-2010-0093901, PCT/KR2010/007523), was identified during this testing. Water extracts were then prepared by steeping finely crushed herbs containing the KIOM-MA medical formulation as described previously [18], with some modification. Briefly, $1840.0 \mathrm{~g}$ of the KIOMMA formula were placed in $18.4 \mathrm{~L}$ of distilled water and boiled for about three hours at $115^{\circ} \mathrm{C}$. The extract was then filtered through a testing sieve (Aperture $500 \mu \mathrm{m}$ and $150 \mu \mathrm{m})$. All herbal plant materials were purchased from Yeongcheon Oriental Herbal Market, Gyeongbuk Province, Republic of Korea.

Fermentation was conducted using Lactobacillus acidophilus (KFRI 128, KCTC 2182) donated from the Korea Food Research Institute (KFRI). Briefly, KIOM-MA water extract was autoclaved for $5 \mathrm{~min}$, after which the $\mathrm{pH}$ was neutralized using the addition of $1 \mathrm{M} \mathrm{NaOH}$. L. acidophyllus was prepared by culturing in MRS broth at $37^{\circ} \mathrm{C}$ under anaerobic conditions. The initial concentration of bacteria ranged from $10^{5}$ to $10^{7} \mathrm{CFU} / \mathrm{mL}$ depending on the substrate. KIOM-MA containing L. acidophyllus was fermented at $37^{\circ} \mathrm{C}$ for $48 \mathrm{~h}$ using MRS medium $(10.0 \mathrm{~g} / \mathrm{L}$ Peptone, $10.0 \mathrm{~g} / \mathrm{L}$ Beef extract, $5.0 \mathrm{~g} / \mathrm{L}$ Yeast extract, $20.0 \mathrm{~g} / \mathrm{L}$ Glucose, $1.0 \mathrm{~mL} / \mathrm{L}$ Tween 80, $2.0 \mathrm{~g} / \mathrm{L} \mathrm{K}_{2} \mathrm{HPO}_{4}, 5.0 \mathrm{~g} / \mathrm{L}$ Sodium acetate, $2.0 \mathrm{~g} / \mathrm{L}$ Triammonium citrate, $0.2 \mathrm{~g} / \mathrm{L} \mathrm{MgSO}_{4} \cdot 7 \mathrm{H}_{2} \mathrm{O}, 0.2 \mathrm{~g} / \mathrm{L}$ $\mathrm{MnSO}_{4} \cdot 4 \mathrm{H}_{2} \mathrm{O}, \mathrm{pH}$ 6.2-6.6). The KIOM-MA128 was then passed through a $60 \mu \mathrm{m}$ nylon net filter (Millipore, MA, USA), precipitated overnight, lyophilized (supernatant), and stored in desiccators at room temperature prior to use.

\subsection{HPLC Analysis of KIOM-MA and KIOM-MA128. Active} principles separation was conducted using a reverse-phase HPLC system consisting of an HPLC pump, as previously described [19]. Fermented samples were monitored at $254 \mathrm{~nm}$ and the area responses were integrated. The major active compounds, isoflavones, were identified based on the retention time and the PDA spectra of the pre- and postfermentation herbal extracts were compared. Standard molecules (liquiritin, nodakenin, icariin, and decursin) were purchased from the Korea Food \& Drug Administration. Glycyrrhizin was purchased from Tokyo Chemical Industry Co. (Tokyo, Japan). Decursinol angelate was purchased from Natural Product Chemistry BioTech Inc. HPLC grade
TABLE 1: Mobile phase condition of chromatographic separation $(254 \mathrm{~nm})$.

\begin{tabular}{lccc}
\hline $\begin{array}{l}\text { Time } \\
(\mathrm{min})\end{array}$ & $\begin{array}{c}\text { Water } \\
\text { (in 2\% acetic acid) }\end{array}$ & $\begin{array}{c}\text { Acetonitrile } \\
\text { (in 2\% acetic acid) }\end{array}$ & $\begin{array}{c}\text { Flow rate } \\
(\mathrm{mL} / \mathrm{min})\end{array}$ \\
\hline 0 & 95 & 5 & 1 \\
5 & 95 & 5 & 1 \\
60 & 20 & 80 & 1 \\
70 & 20 & 80 & 1 \\
75 & 95 & 5 & 1 \\
90 & 95 & 5 & 1 \\
\hline
\end{tabular}

solutions, water, acetonitrile, and glacial acetic acid were purchased from J. T. Baker Co. (NJ, USA).

2.3. Chromatographic Conditions. The high-performance liquid chromatography (HPLC) data were obtained using an Elite Lachrom analytical HPLC PDA system that included an L-2130 binary HPLC pump, an L-2200 autosampler, a column oven (L-2350), and a diode array UV/VIS detector (L2455). The output signal of the detector was recorded using the EZchrom Elite software for Hitachi. For separation of the samples, an OptimaPak C 18 column $(4.6 \mathrm{~mm} \times 250 \mathrm{~mm}$, $5 \mu \mathrm{m}$, RS tech, republic of Republic of Korea) was employed and the PDA UV wavelength was $254 \mathrm{~nm}$. The mobile phase was water and acetonitrile with a gradient elution containing $2 \%$ glacial acetic acid at a flow rate of $1.0 \mathrm{~mL} / \mathrm{min}$. The column temperature was maintained at $40^{\circ} \mathrm{C}$ (Table 1 ) and the injection volume of the samples was $10 \mu \mathrm{L}$.

2.4. Preparation of Standard Solutions and Samples. To prepare the analytical samples, KIOM-MA and KIOM-MA128 (fermented KIOM-MA) powder were accurately weighed and dissolved in $100 \% \mathrm{H}_{2} \mathrm{O}$ at a concentration of $40 \mathrm{mg} / \mathrm{mL}$. Prior to analysis, the sample was filtered through a $0.45 \mu \mathrm{m}$ filter.

2.5. Animals. 3-week-old male BALB/c mice were obtained from Hanlim Laboratory Animals Co. (Hwasung, KyunggiDo, Korea) and maintained for two weeks before the start of the experiments. All animals were maintained on a standard light-dark cycle at ambient temperature $\left(23 \pm 2^{\circ} \mathrm{C}\right)$ and humidity $(55 \pm 10 \%)$ with free access to chow pellets and water. The experimental groups, which consisted of 5-7 animals per drug and dose, were selected by a randomized schedule. Animal treatment and maintenance were conducted in accordance with the Principle of Laboratory Animal Care (NIH publication no. 85-23 revised in 1985) and the Animal Care and Use Guidelines of Sahmyook University, Republic of Korea.

2.6. Atopic Dermatitis Models. 5-week-old BALB/c mice were anesthetized with ether and the dorsal skin was then shaved with a clipper and a shaver one day before experiments. The exposed dorsal region was treated with Ovalbumin and aluminum hydroxide to induce AD. Mice were randomly assigned to one of seven groups at the start 


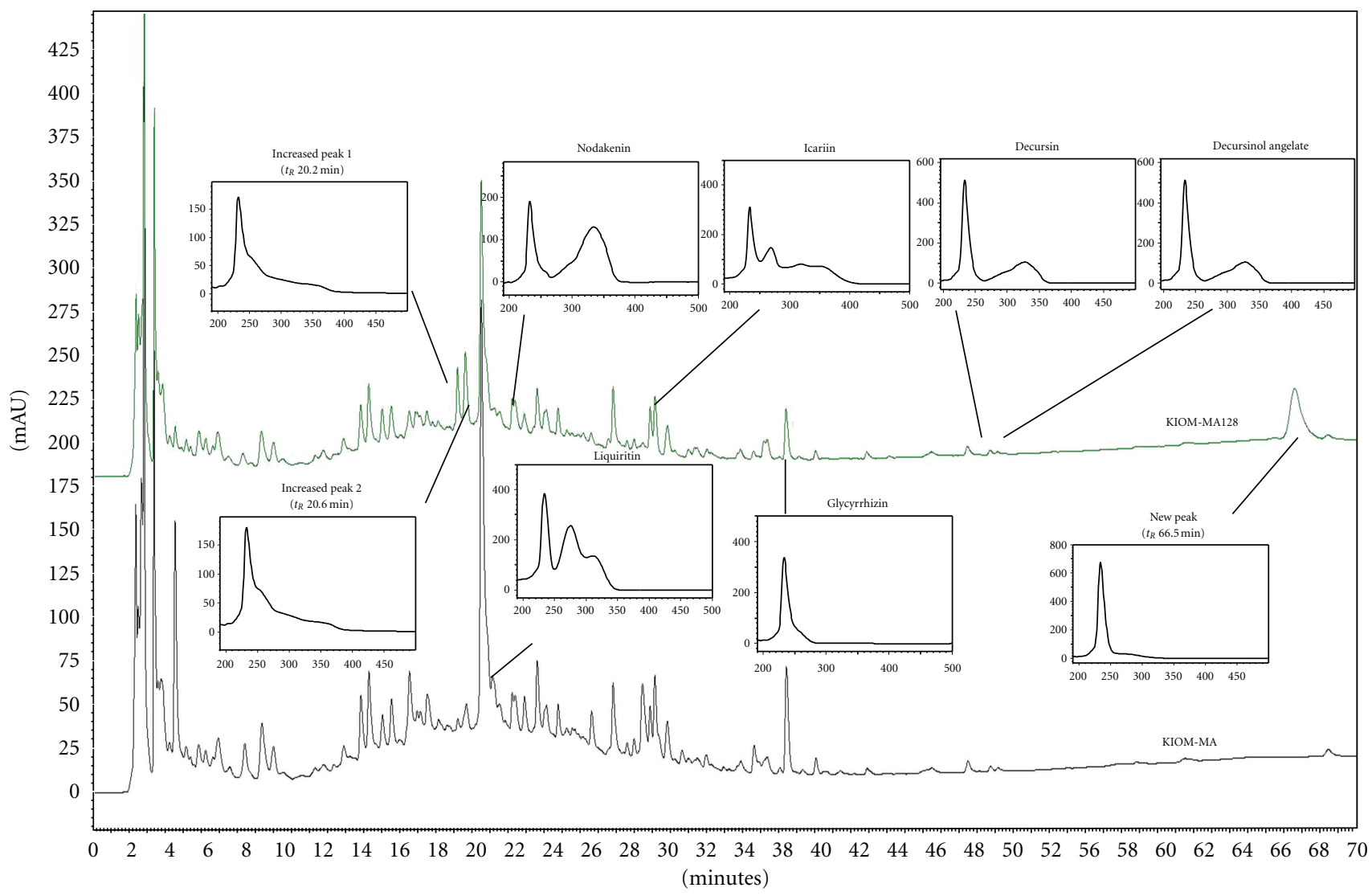

FIgure 1: The HPLC chromatogram of KIOM-MA and KIOM-MA128 at $254 \mathrm{~nm}$.

of the experiment $(n=5)$ : Group A, normal control; Group B, AD control; Group C, KIOM-MA treatment (50 mg/kg); Group D, KIOM-MA treatment $(100 \mathrm{mg} / \mathrm{kg})$; Group E, KIOM-MA-128 treatment (50 mg/kg); Group F, KIOM-MA128 treatment $(100 \mathrm{mg} / \mathrm{kg})$; and Group $\mathrm{G}$, dexamethasone (1 mg/kg). Following the last administration of KIOM-MA or KIOM-MA128 on day 14, the mice were sacrificed and the plasma IgE levels were measured.

2.7. Clinical Skin Severity Score. The dorsal skin of each mouse was photographed before, during, and after KIOMMA or KIOM-MA128 treatment. The severity of AD-like dorsal skin lesions was assessed once a day as follows: dorsal lesions were evaluated for five symptoms, erythema/darkening, edema/papulation, excoriations, lichenification/prurigo, and dryness. Each symptom was graded from 0 to 3 (none, 0 ; mild, 1 ; moderate, 2 ; severe, 3 ). The clinical skin score was defined as the sum of the individual scores, ranging from 0 to 15 .

2.8. Established Lesions and Scratching Behavior. The scratching behavior was recorded on video once a day for 11 consecutive days. Specifically, the number of times a mouse scratched the dorsal skin lesion within a period of 15 min was counted. Because the average number of scratches in each mouse varied daily, the scratching behavior was estimated as the percentage of the control calculated from the mean value of the no-treatment group.

2.9. Detection of Serologic IgE Concentration. Mice were sacrificed on 1, 3, 7, and 14 days after treatment of KIOM-MA or KIOM-MA128, and cardiac blood was collected from mice by thoracotomy into EDTA-treated tubes, after which plasma was separated by centrifugation at $3,000 \mathrm{~g}$ for $10 \mathrm{~min}$ at $4^{\circ} \mathrm{C}$ and stored at $-80^{\circ} \mathrm{C}$. The plasma levels of $\operatorname{IgE}$ were measured with ELISA kits ( R \& D System, Boston, MA, USA) according to the manufacturer's instruction.

2.10. Statistical Analysis. Experimental values are given as the means \pm SEM. The statistical difference was determined by a two-sided Mann-Whitney $U$-test. A $P<0.05$ was considered to indicate statistical significance.

\section{Results}

3.1. HPLC Analysis. Six maker compounds of KIOMMA and KIOM-MA128 liquiritin (tR $22.1 \mathrm{~min}$ ), nodakenin ( $\mathrm{tR} 23.4 \mathrm{~min}$ ), icariin ( $\mathrm{tR} 31.1 \mathrm{~min}$ ), glycyrrhizin ( $\mathrm{tR}$ $38.4 \mathrm{~min}$ ), decursin ( $\mathrm{tR} 49.7 \mathrm{~min}$ ), and decursinol angelate (tR $50.1 \mathrm{~min}$ ) were identified by HPLC PDA analysis and comparison with standard compounds. Liquiritin ( $\mathrm{tR} 22.1 \mathrm{~min}$ ), icariin ( $\mathrm{tR} 31.1 \mathrm{~min}$ ), and glycyrrhizin 


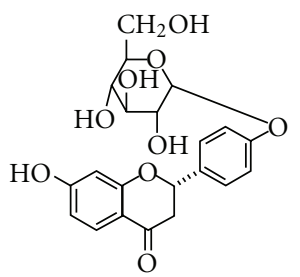

Liquiritin

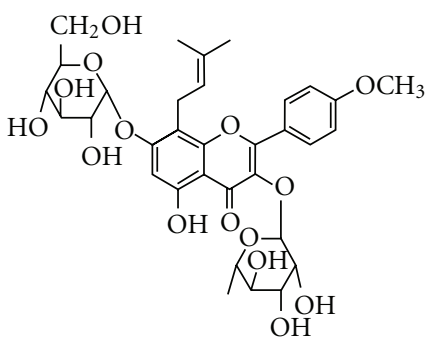

Icariin<smiles>CC1=CC(=O)OC(C2Cc3cc4ccc(=O)oc4cc3OC2(C)C)C1</smiles>

Decursin

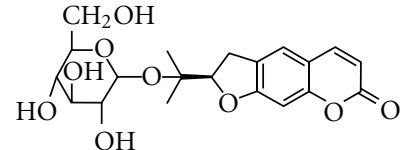

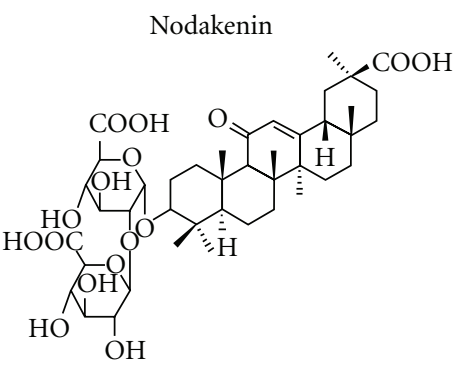<smiles>C=C(C)C(=O)OC1Cc2cc3ccc(=O)oc3cc2OC1(C)C</smiles>

Decursinol angelate

FIgURE 2: The chemical structure of six markers in KIOM-MA and KIOM-MA128.

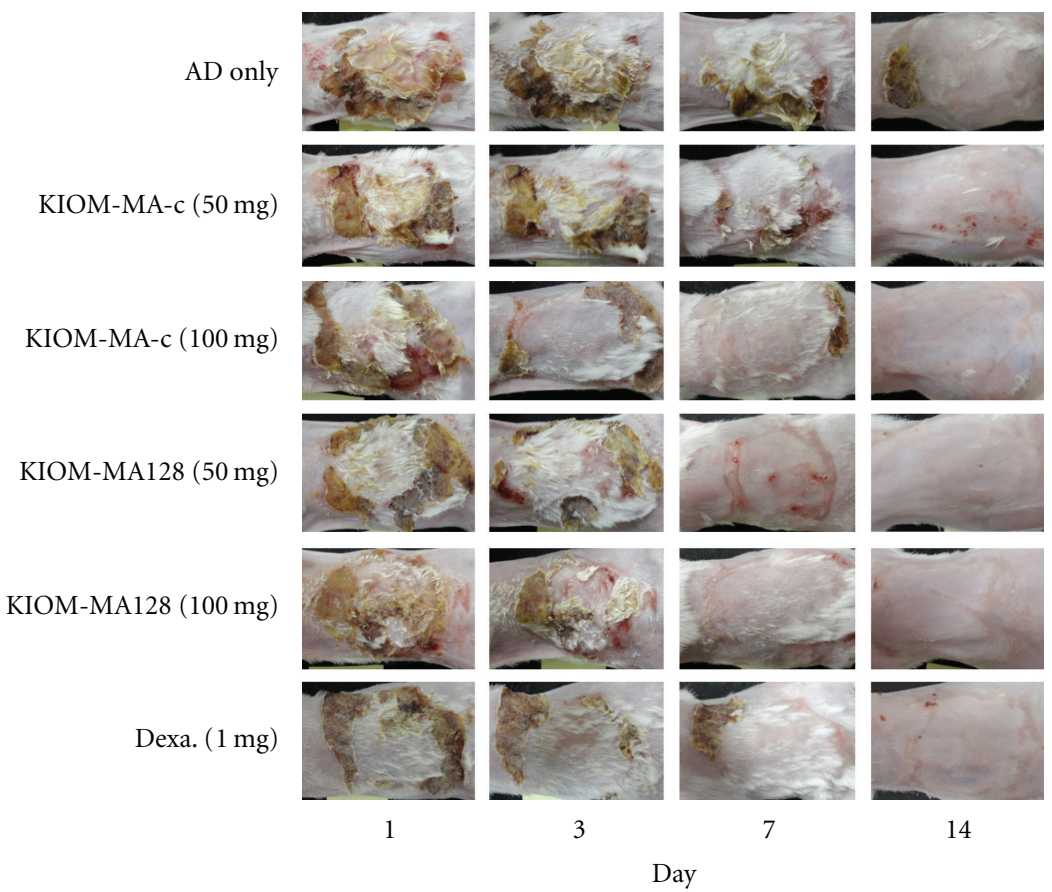

FIgURE 3: The effect of KIOM-MA and KIOM-MA128 on atopic dermatitis-induced BALB/c mice. KIOM-MA and KIOM-MA128 significantly mitigated five symptoms: erythema/darkening, edema/population, excoriations, lichenification/prurigo, and dryness at 7 days after treatment. 


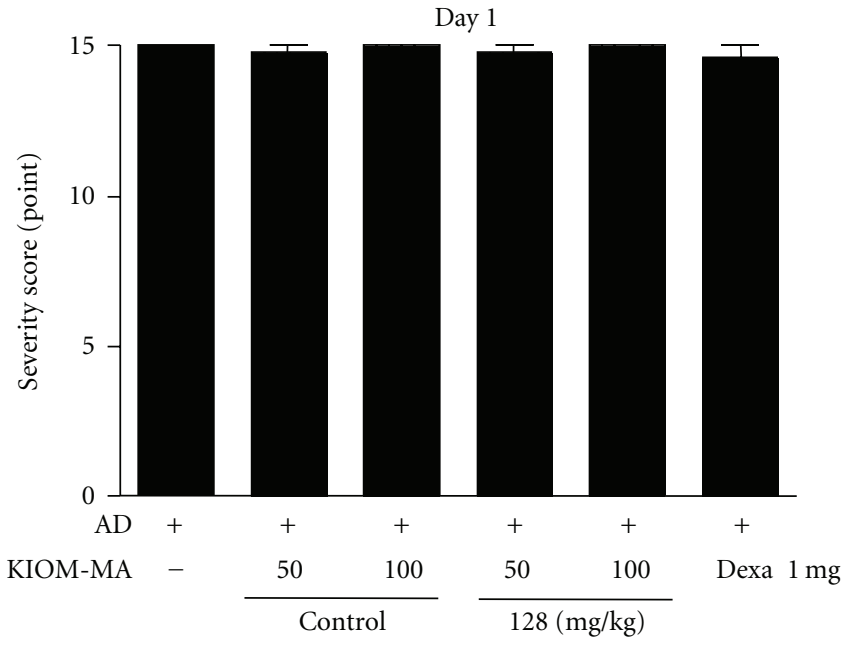

(a)

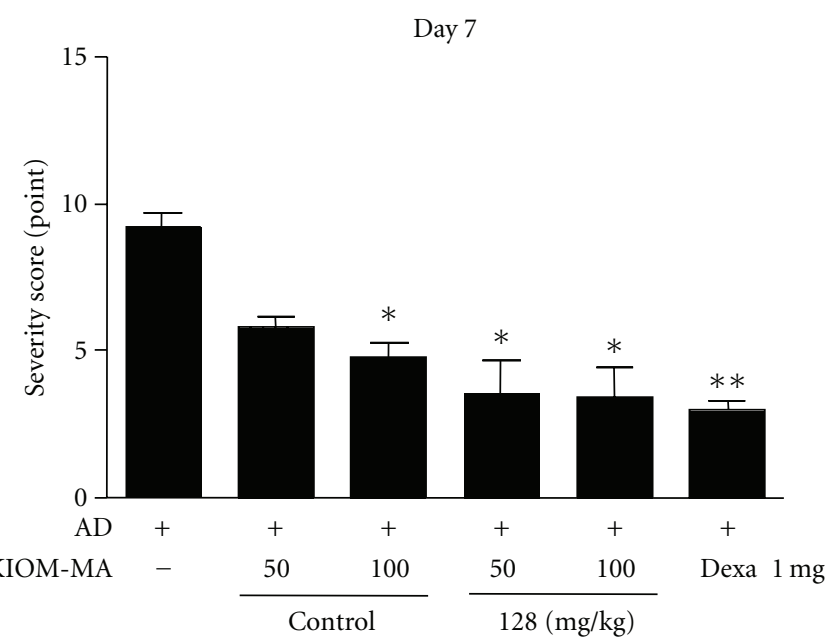

(c)

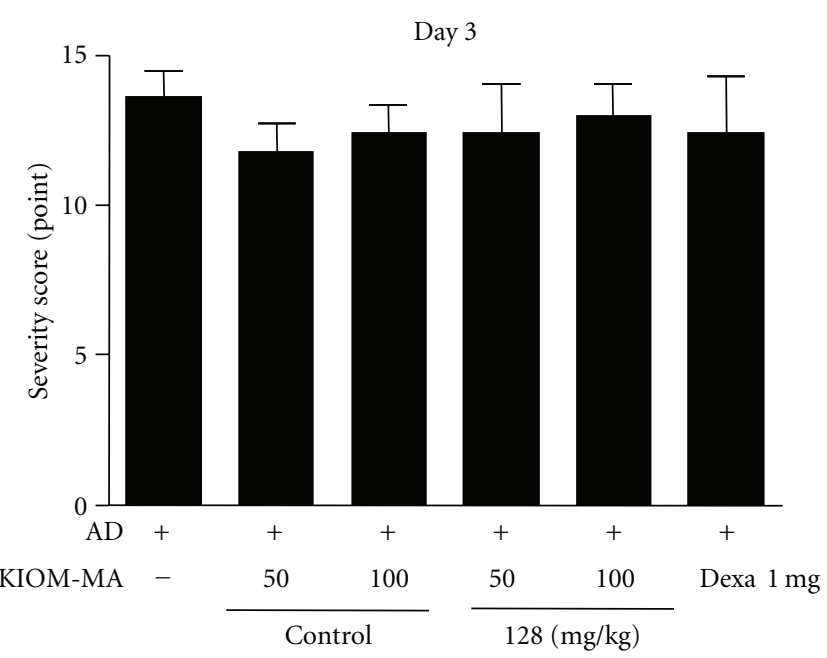

(b)

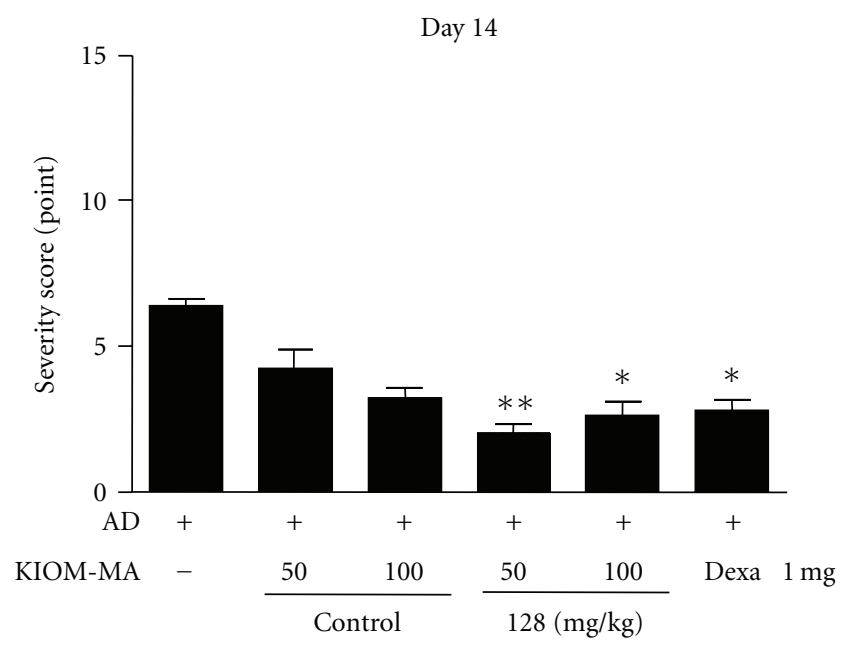

(d)

FIgURE 4: The effect of KIOM-MA and KIOM-MA128 on skin lesions. The improvement in skin lesions was evaluated based on the skin severity score. The clinical skin severity was decreased in the KIOM-MA-treated group and much more improved in KIOM-MA128 after 7 days. Experimental values are given as the means \pm SEM $(n=5) .{ }^{*} P<0.05$, ${ }^{* *} P<0.01$, when compared with untreated group.

(tR $38.4 \mathrm{~min}$ ) were lower in KIOM-MA128 (fermented KIOM-MA) following deglycosylation during fermentation. Two peaks at retention times of 20.2 and 20.6 minutes increased and one peak at 66.5 minutes appeared. The structures of the two increased constituents and the new constituent were identified by chromatographic separation and spectroscopic techniques (Figures 1 and 2).

\subsection{KIOM-MA and KIOM-MA128 Exhibited Potent Anti-} atopic Effect in BALB/c Mice Atopic Dermatitis Model. To investigate the effect of KIOM-MA and KIOM-MA128 on $\mathrm{AD}$, 50, or 100 mg KIOM-MA, KIOM-MA128, 1 mg dexamethasone or a control (without KIOM additive) was orally administered to five-week-old BALB/c mice, which have AD induced by ovalbumin and aluminum hydroxide, once a day for consecutive days. The atopic lesions of mice were observed until 14 days after the first treatment. From 7 days posttreatment, both KIOM-MA and KIOM-MA128 remarkably improved atopic symptoms comparable to control dexamethasone, which is well-known anti-inflammatory reagent (Figure 3 ). Improved five symptoms contain erythema/darkening, edema/papulation, excoriations, lichenification/prurigo, and dryness.

\subsection{The Effect of KIOM-MA and KIOM-MA128 on Clinical} Skin Severity Score. Next, the improvement observed in atopic skin lesions by KIOM-MA and KIOM-MA128 was evaluated based on the skin severity score. The clinical skin severity score was calculated from five major symptoms consisting of symptomerythema/darkening, edema/papulation, excoriations, lichenification/prurigo, and dryness. As shown in Figure 4, skin severity score was significantly decreased in the KIOM-MA-treated group $(P$ value $=0.0422$ and 0.0487 at 50 and $10 \mathrm{mg} / \mathrm{kg}$, resp., compared with no-treated group) and much more reduced in KIOM-MA128 ( $P$ value $=0.0282$ and 0.0120 at 50 and $10 \mathrm{mg} / \mathrm{kg}$, resp., compared 


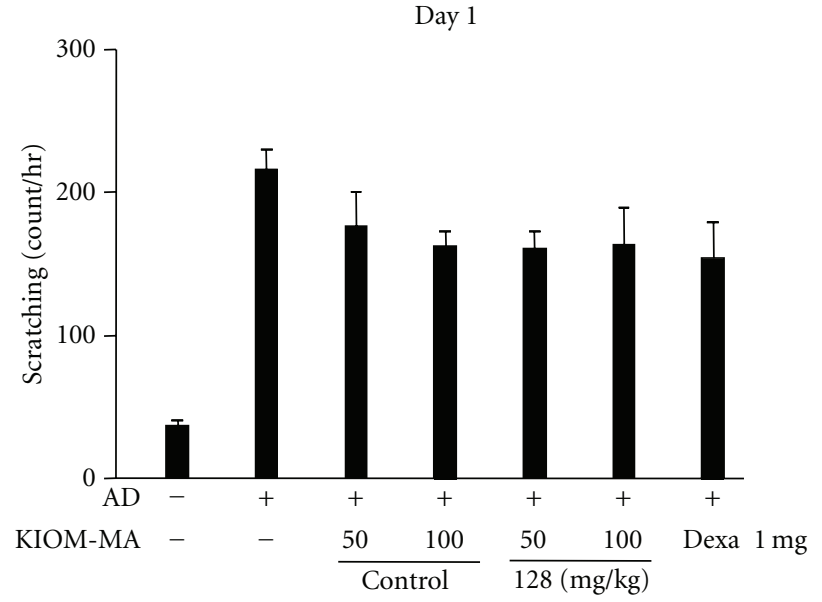

(a)

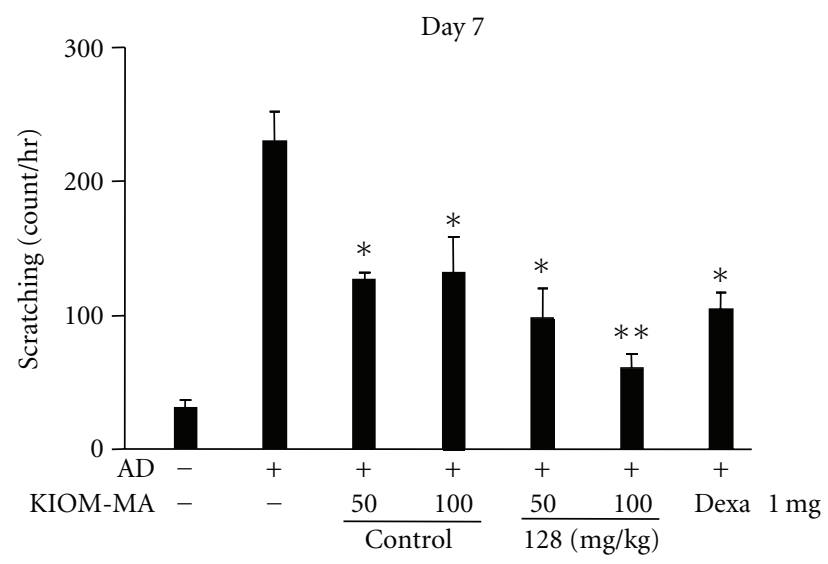

(c)

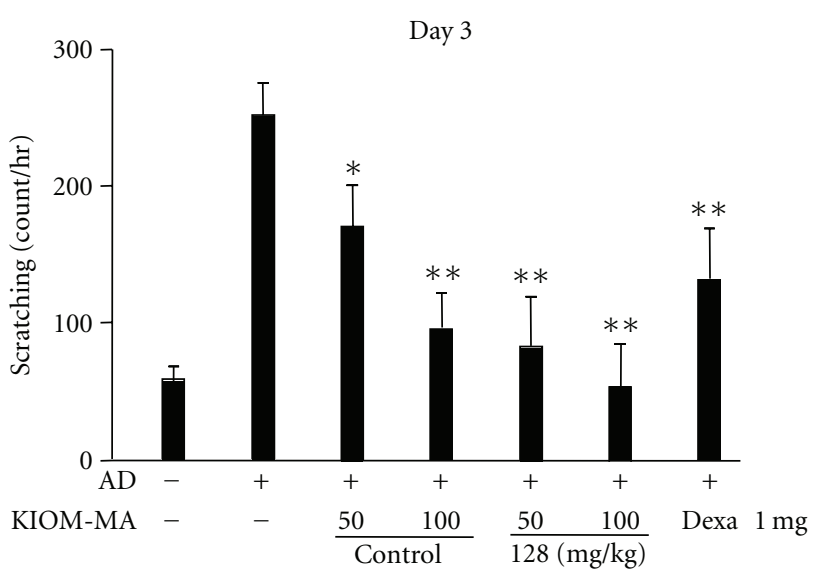

(b)

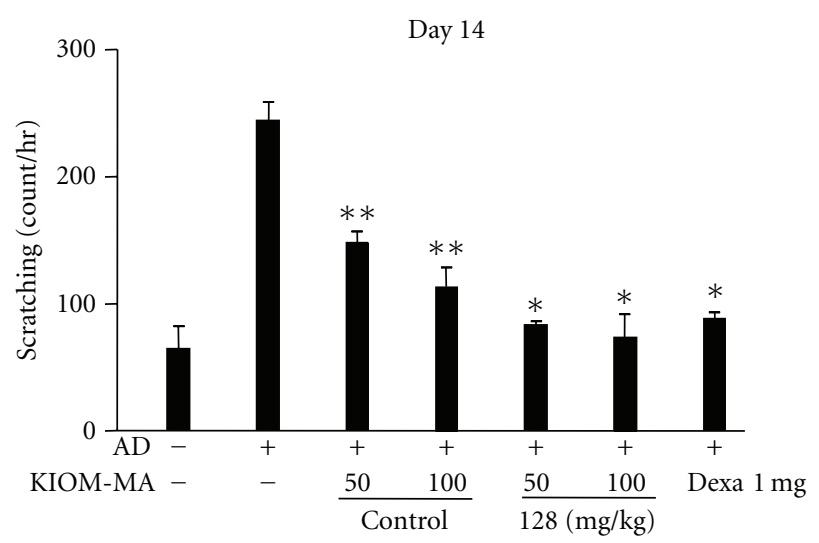

(d)

FIGURE 5: The effect of KIOM-MA and KIOM-MA128 on scratching behavior. KIOM-MA or KIOM-MA128 was administered to the mice as described in the Materials and Methods. Simultaneously, we counted the number of times the mice scratched skin lesions for 30 min on 14 consecutive days. KIOM-MA and KIOM-MA128 caused a decrease in the scratching behavior, which was accompanied by improvement of skin eruptions. ${ }^{*} P<0.05,{ }^{* *} P<0.01$, when compared with untreated group.

with no-treated group) after 7 days. KIOM-MA and KIOMMA128 tended to decrease the skin severity score on day 5, and the scores of KIOM-MA and KIOM-MA128 were 5 and 3 , respectively, compared with that of negative control that was 10 on day 7 (Figure 4). The skin severity score of dexamethasone was 3, same extent with KIOM-MA128. On day 14, the scores of all groups were decreased, and on day 21, KIOM-MA or dexamethasone-treated group had the same clinical skin score as the no-treatment and the control group (data not shown). Whether to determine if the effect of KIOM-MA or KIOM-MA128 depended on the treatment dose, we administered different doses (50 and $100 \mathrm{mg} / \mathrm{kg}$ ) of KIOM-MA. We found that both KIOM-MA and KIOM-MA128 reduce the clinical skin severity score in a dose-dependent manner on day 7 (Figure 4).

3.4. The Effect of KIOM-MA and KIOM-MA128 on Scratching Behavior. We next investigated the effects of oral treatment of KIOM-MA on the scratching behavior in the mice. As represented in Figure 5, KIOM-MA reduced the number of scratches and KIOM-MA128 exhibited more significantly decreased scratches on day 3 after the start of treatment, which was accompanied by an improvement in the eruptions (data not shown). On day 7 after treatment, the numbers of scratching in atopic mice by treatment of KIOM-MA or KIOM-MA128 were significantly lower than control dexamethasone. The diminished scratching behavior by treatment of KIOM-MA ( $P$ value $=0.0197$ and 0.02368 at 50 and $10 \mathrm{mg} / \mathrm{kg}$, resp., compared with no-treated group) or KIOMMA128 $(P$ value $=0.0028$ and 0.0013 at 50 and $10 \mathrm{mg} / \mathrm{kg}$, resp., compared with no-treated group) was observed in dose-dependent manner. An increase in the scratching behavior of the mice was again observed immediately after the discontinuation of the KIOM-MA treatment (data not shown).

3.5. The Effect of KIOM-MA and KIOM-MA128 on IgE Secretion. Because $\mathrm{AD}$ is a type I IgE-mediated hypersensitivity reaction contributing to immune dysregulation and its major characteristics is hyperproduction of IgE, we examined the 


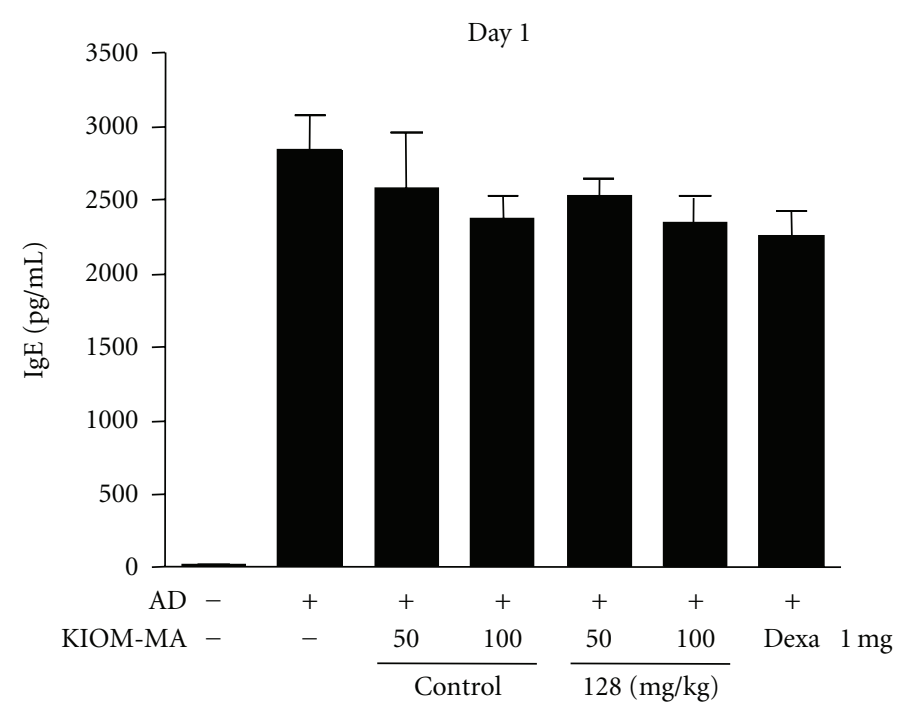

(a)

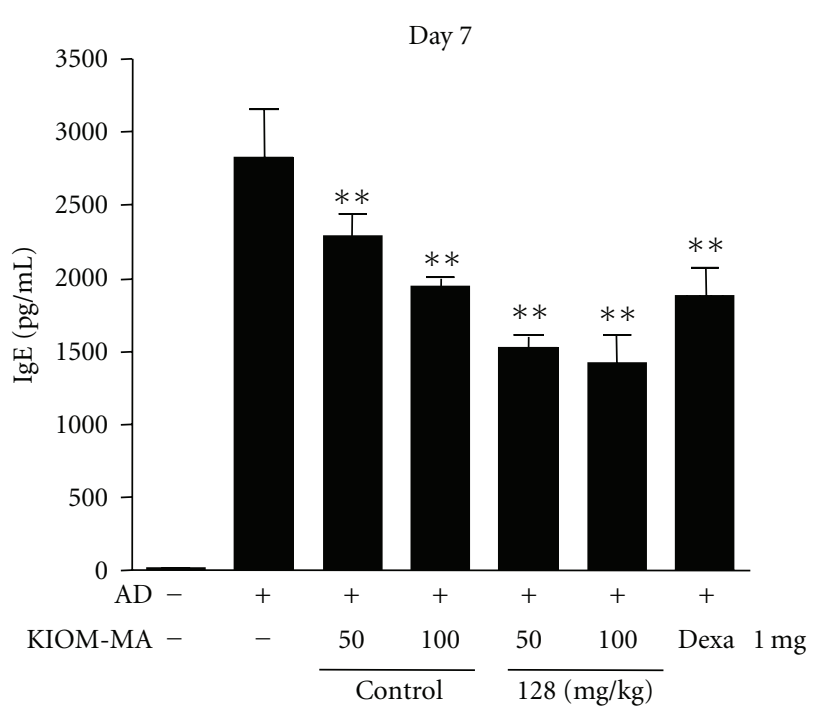

(b)

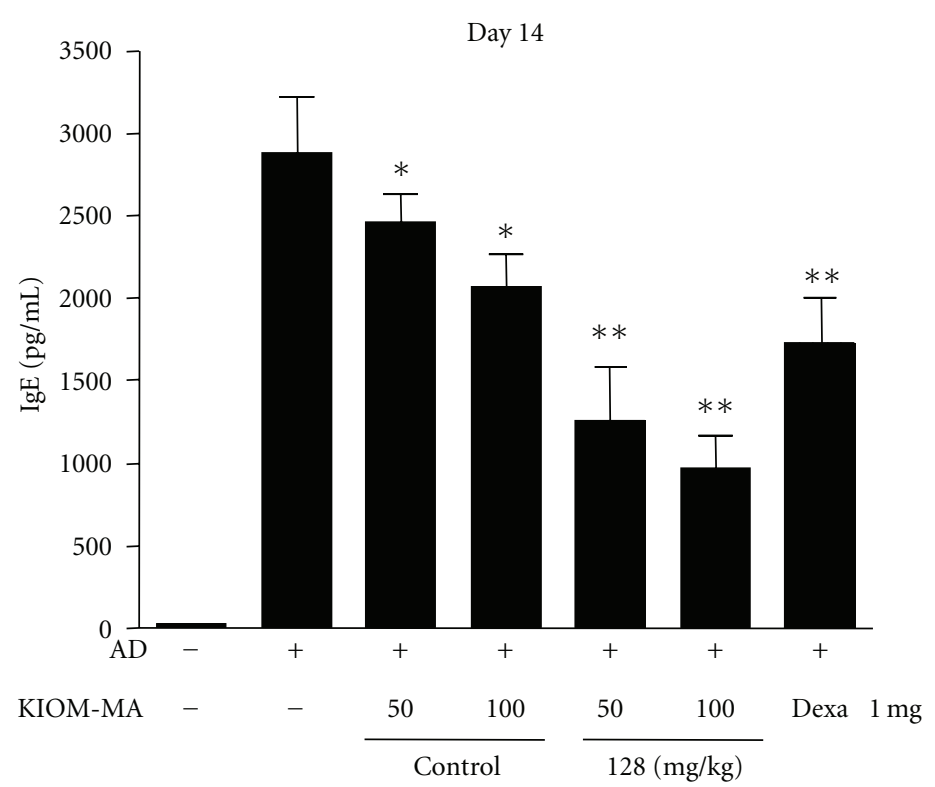

(c)

Figure 6: The effect of KIOM-MA and KIOM-MA128 on IgE secretion. A drastic decrease in the IgE levels was observed in KIOM-MA and KIOM-MA128-treated mice. Values are the mean $\pm \operatorname{SEM}(n=5) * P<0.05$, ${ }^{* *} P<0.01$, when compared with the no-treatment group.

plasma IgE levels in the mice treated with KIOM-MA or KIOM-MA128 and compared with that of control. Figure 6 shows that when the mice were induced to the $\mathrm{AD}$, the plasma IgE concentration was highly increased than control group, and the IgE levels increased in the $\mathrm{AD}$ mice were reduced with KIOM-MA $(P$ value $=0.0090$ and 0.0051 at 50 and $10 \mathrm{mg} / \mathrm{kg}$, resp., compared with no-treated group) or KIOM-MA128 $(P$ value $=0.0019$ and 0.0023 at 50 and $10 \mathrm{mg} / \mathrm{kg}$, resp., compared with no-treated group) treatment from day 7 after treatment. Consistent with skin severity and starching behavior results, KIOM-MA128 exhibited much stronger reducing effect on IgE secretion in the plasma of AD mice model (Figure 6).

\section{Discussion}

We investigated the effects of KIOM-MA and its fermented product, KIOM-MA128, in vitro and in vivo in an AD-like animal model. The results demonstrated that KIOM-MA effectively reduced the clinical features and the index of $\mathrm{AD}$ like serum IgE level. Considering that AD is the most common skin disease, it is worth noting that the clinical recovery from AD-like skin was more clearly seen based on macrography, scratching count, and severity scores in the treatment group. Hyperpigmentations and erythematous lesion eruptions also gradually improved significantly in the treatment group in a dose-dependent manner. The mice treated with 
KIOM-MA or KIOM-MA128 did not show any clinical signs in the skin after seven days of oral administration comparable to dexamethasone-treated group (positive control group). The no-treatment group suffered continuously from skin eruptions and skin injuries in response to scratching behavior after seven days. Furthermore, KIOM-MA and KIOM-MA128 significantly inhibited the AD clinical signs in a dose-dependent manner. However, topical application routes for atopic therapy are needed and are being tested now. The pharmacodynamics and action mechanisms of KIOM-MA and KIOM-MA128 are currently being investigated in vitro with a focus on Th1/Th2 cytokines and related chemokines.

Several previous studies have evaluated the use of glycyrrhizin [20], liquiritin [21], and decursin [21] for the treatment of $\mathrm{AD}$, as well as nodakenin for anti-inflammatory effects [22], icariin for immunoregulatory effects [23], and decursinol angelate for blocking inflammatory activation [24]. Our results indicate that the high concentration of several constituents including glycyrrhizin contained in the KIOMMA may be responsible for anti-inflammatory effect of KIOM-MA and KIOM-MA128. Interestingly, the KIOMMA128 fermented from KIOM-MA by L. acidophilus more significantly improved atopic symptoms. Fermentation, a progress of decomposition of organic matter occurred by L. acidophilus, generates various low molecular weight substances like aglycone from macromolecule including glycoside. This fact implies that active aglycone formed during fermentation is more infiltrative than glycoside in vivo. Aglycones may increase the skin or mucosal permeability, thus, optimizing the physiological bioavailability in vivo. In addition, HPLC-DAD analysis of KIOM-MA and KIOMMA128 strongly implies that new constituents or increased constituents may be involved in the antiallergic mechanism. However, further studies should be performed to test these hypotheses. Two increased peaks and one new peak are now being subjected to isolate for the further study.

In summary, the pharmacological profiles of KIOM-MA and KIOM-MA128 were as follows: the reduction of clinical features and IgE production suggest that KIOM-MA and KIOM-MA128 are effective for the treatment of AD in a dose-dependent manner. The oral administration route may be useful for clinical improvement of $\mathrm{AD}$, even though topical application is generally thought to have more therapeutic advantages. Bioconversion by fermentation significantly enhances the anti-inflammatory effect of KIOMMA. Further studies are necessary to clarify the mechanisms of KIOM-MA and KIOM-MA128 for the treatment of AD. Nevertheless, our findings suggest that KIOM-MA and KIOM-MA128, which has stronger efficacy, could be applicable to develop potent therapeutic reagent for the treatment of AD.

\section{Authors' Contribution}

T. H. Chung and T. J. Kang authors contributed equally to this work.

\section{Acknowledgment}

This study was supported by Grant K11050 awarded to the Korea Institute of Oriental Medicine (KIOM) from the Ministry of Education, Science and Technology (MEST), Republic of Korea.

\section{References}

[1] D. Y. M. Leung, M. Boguniewicz, M. D. Howell, I. Nomura, and Q. A. Hamid, "New insights into atopic dermatitis," Journal of Clinical Investigation, vol. 113, no. 5, pp. 651-657, 2004.

[2] N. Novak, T. Bieber, and D. Y. Leung, "Immune mechanisms leading to atopic dermatitis," Journal of Allergy and Clinical Immunology, vol. 112, no. 6, supplement, pp. S128-S139, 2003.

[3] D. Y. M. Leung, "Pathogenesis of atopic dermatitis," Journal of Allergy and Clinical Immunology, vol. 104, no. 3, supplement, pp. S99-S108, 1999.

[4] J. M. Spergel and A. S. Paller, "Atopic dermatitis and the atopic march," Journal of Allergy and Clinical Immunology, vol. 112, no. 6, supplement, pp. S118-S127, 2003.

[5] P. Juto, S. Engberg, and J. Winberg, "Treatment of infantile atopic dermatitis with a strict elimination diet," Clinical Allergy, vol. 8, no. 5, pp. 493-500, 1978.

[6] R. Sidbury and J. M. Hanifin, "Systemic therapy of atopic dermatitis," Clinical and Experimental Dermatology, vol. 25, no. 7, pp. 559-566, 2000.

[7] U. R. Hengge, T. Ruzicka, R. A. Schwartz, and M. J. Cork, "Adverse effects of topical glucocorticosteroids," Journal of the American Academy of Dermatology, vol. 54, no. 1, pp. 1-15, 2006.

[8] M. Furue, H. Terao, Y. Moroi et al., "Dosage and adverse effects of topical tacrolimus and steroids in daily management of atopic dermatitis," Journal of Dermatology, vol. 31, no. 4, pp. 277283, 2004.

[9] J. M. Hanifin, "Atopic dermatitis: new therapeutic considerations," Journal of the American Academy of Dermatology, vol. 24, no. 6, pp. 1097-1101, 1991.

[10] M. P. Sheehan, M. H. A. Rustin, D. J. Atherton et al., "Efficacy of traditional Chinese herbal therapy in adult atopic dermatitis," The Lancet, vol. 340, no. 8810, pp. 13-17, 1992.

[11] T. M. Nagle, S. M. Torres, K. L. Horne, R. Grover, and M. T. Stevens, "A randomized, double-blind, placebo-controlled trial to investigate the efficacy and safety of a Chinese herbal product (P07P) for the treatment of canine atopic dermatitis," Veterinary Dermatology, vol. 12, no. 5, pp. 265-274, 2001.

[12] K. L. Hon, T. F. Leung, P. C. Ng et al., "Efficacy and tolerability of a Chinese herbal medicine concoction for treatment of atopic dermatitis: a randomized, double-blind, placebo-controlled study," British Journal of Dermatology, vol. 157, no. 2, pp. 357-363, 2007.

[13] R. Yuan and Y. Lin, "Traditional Chinese medicinean approach to scientific proof and clinical validation," Pharmacology \& Therapeutics, vol. 86, no. 2, pp. 191-198, 2000.

[14] W. Zhang, T. Leonard, F. Bath-Hextall et al., "Chinese herbal medicine for atopic eczema," Cochrane Database of Systematic Reviews, vol. 2, Article ID CD002291, 2005.

[15] D. M. Marcus and A. P. Grollman, "Botanical medicines - The need for new regulations," The New England Journal of Medicine, vol. 347, no. 25, pp. 2073-2076, 2002. 
[16] J. B. Calixto, "Efficacy, safety, quality control, marketing and regulatory guidelines for herbal medicines (phytotherapeutic agents)," Brazilian Journal of Medical and Biological Research, vol. 33, no. 2, pp. 179-189, 2000.

[17] S. S. Joo, T. J. Won, S. Y. Nam et al., "Therapeutic advantages of medicinal herbs fermented with Lactobacillus plantarum, in topical application and its activities on atopic dermatitis," Phytotherapy Research, vol. 23, no. 7, pp. 913-919, 2009.

[18] J. D. van der Merwe, E. Joubert, E. S. Richards et al., "A comparative study on the antimutagenic properties of aqueous extracts of Aspalathus linearis (rooibos), different Cyclopia spp. (honeybush) and Camellia sinensis teas," Mutation Research, vol. 611, no. 1-2, pp. 42-53, 2006.

[19] J. Chun, G. M. Kim, K. W. Lee et al., "Conversion of isoflavone glucosides to aglycones in soymilk by fermentation with lactic acid bacteria," Journal of Food Science, vol. 72, no. 2, pp. M39M44, 2007.

[20] M. Saeedi, K. Morteza-Semnani, and M. R. Ghoreishi, "The treatment of atopic dermatitis with licorice gel," Journal of Dermatological Treatment, vol. 14, no. 3, pp. 153-157, 2003.

[21] J. H. Kim, J. H. Jeong, S. T. Jeon et al., "Decursin inhibits induction of inflammatory mediators by blocking nuclear factor- $\kappa \mathrm{B}$ activation in macrophages," Molecular Pharmacology, vol. 69, no. 6, pp. 1783-1790, 2006.

[22] T. Yoon, D. Y. Lee, A. Y. Lee, G. Choi, B. K. Choo, and H. K. Kim, "Anti-inflammatory effects of Glehnia littoralis extract in acute and chronic cutaneous inflammation," Immunopharmacology and Immunotoxicology, vol. 32, no. 4, pp. 663-670, 2010.

[23] W. He, H. Sun, B. Yang, D. Zhang, and D. Kabelitz, "Immunoregulatory effects of the Herba Epimediia glycoside icariin," Arzneimittel-Forschung, vol. 45, no. 8, pp. 910-913, 1995.

[24] W. J. Kim, M. Y. Lee, J. H. Kim, K. Suk, and W. H. Lee, “Decursinol angelate blocks transmigration and inflammatory activation of cancer cells through inhibition of PI3K, ERK and NF$\kappa \mathrm{B}$ activation," Cancer Letters, vol. 296, no. 1, pp. 35-42, 2010. 


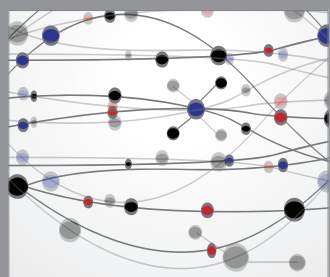

The Scientific World Journal
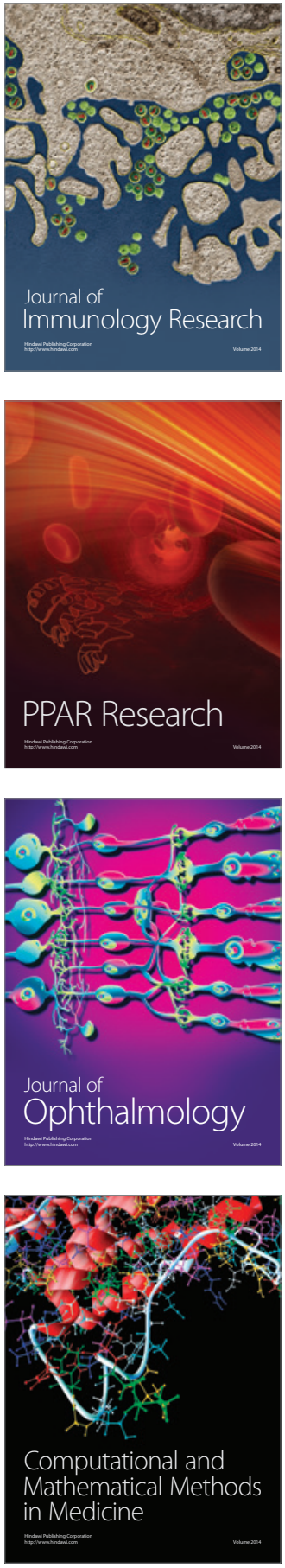

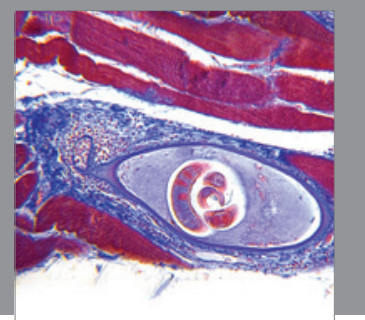

Gastroenterology

Research and Practice
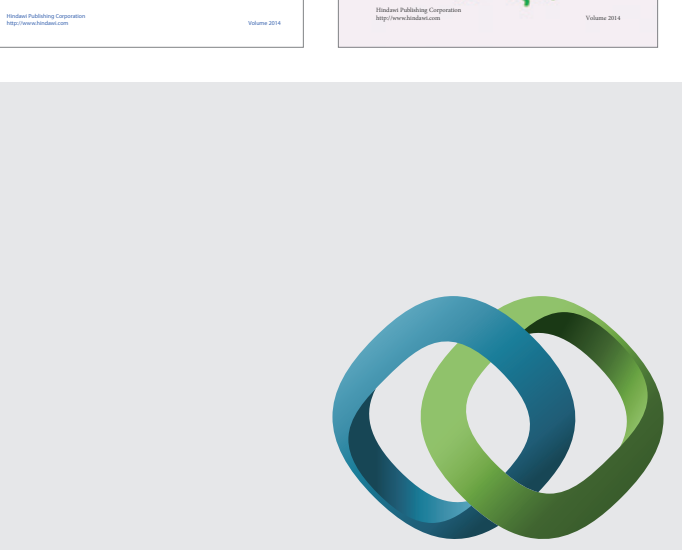

\section{Hindawi}

Submit your manuscripts at

http://www.hindawi.com
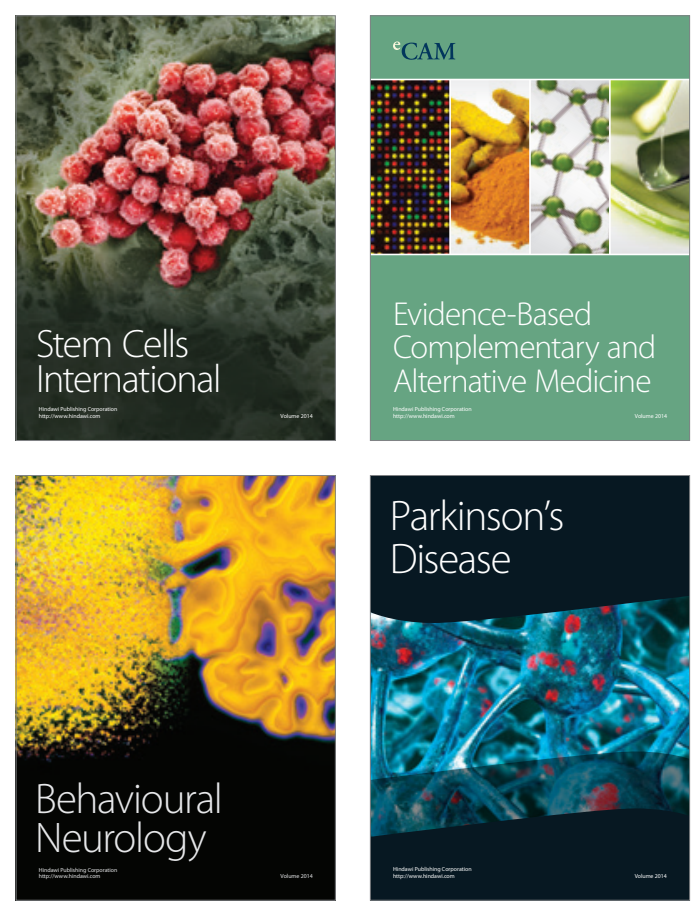

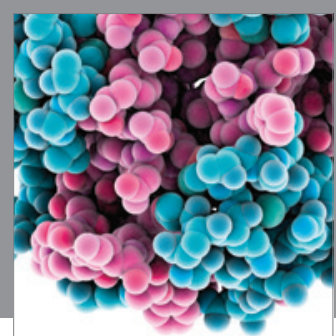

Journal of
Diabetes Research

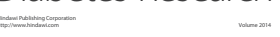

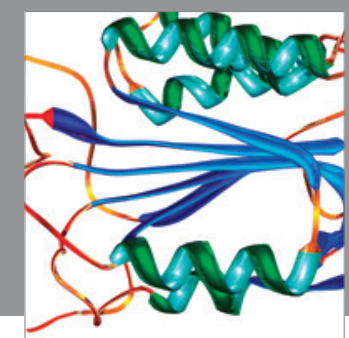

Disease Markers
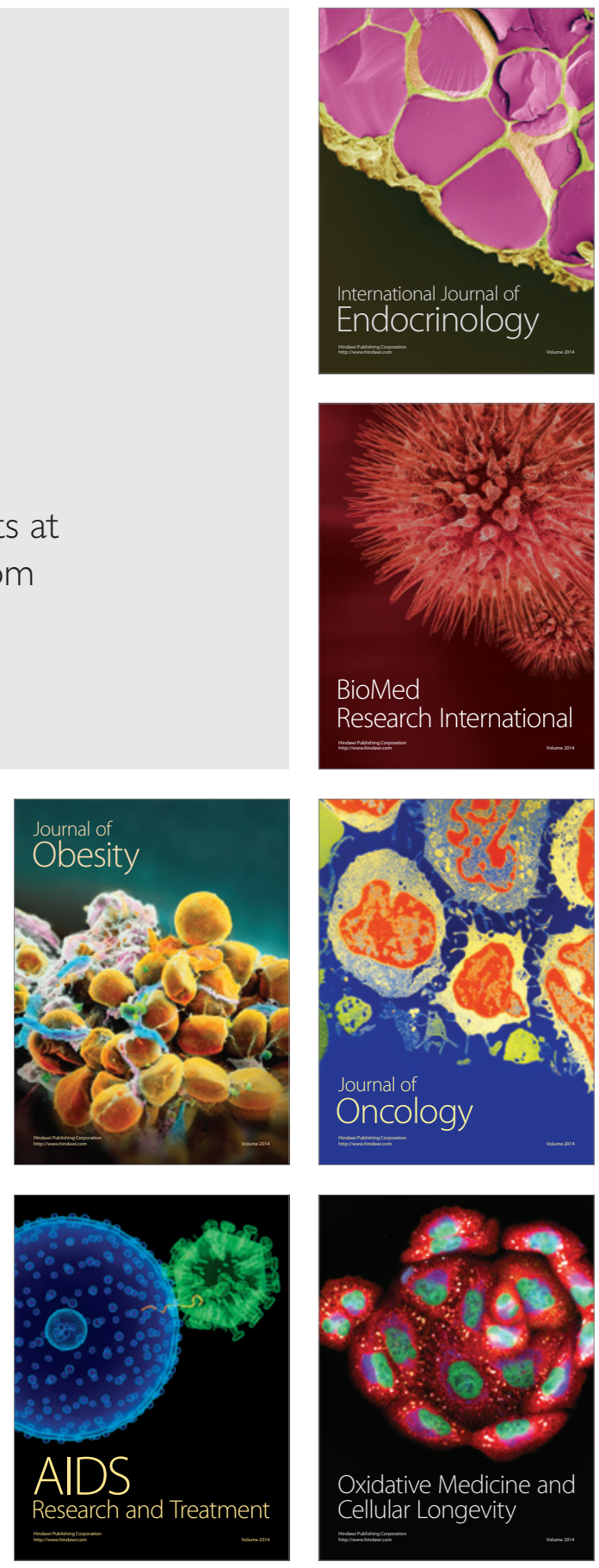